\title{
Mutational spectrum of tobacco associated oral squamous carcinoma and its therapeutic significance
}

\author{
Nishant Batta and Manoj Pandey ${ }^{*}$ (I)
}

\begin{abstract}
Oral squamous cell cancer (OSCC) is a common malignancy attributed to use of chewing smokeless tobacco and smoking. Most of the targeted strategies are based on EGFR expression and mutation; however, none of them has shown significant improvement in survival and response rates. We carried out this study to evaluate mutational profile of tobacco associated oral carcinoma with special emphasis on EGFR and its downstream events.
\end{abstract}

Patients and methods: A total of 46 histologically proven cases were recruited between January 2017 and January 2019. Apart from detailed clinical and histological studies, the paraffin-embedded tissue was submitted for expression of 50 genes using Next Generation Sequencing using lon Ampliseq Cancer Hotspot Panel v2.

Results: The mean age of patients was $47.8 \pm 10.9$ years. Majority had tumors on buccal mucosa (24) and tongue (13). Nineteen of these tumors were larger than $4 \mathrm{~cm}$, and 5 had adjacent site involvement. Thirty one were node positive. TP53 mutations were commonest seen in 19 followed by CDKN2A in 11, HRAS in 8, PIK3CA in 3, SMARCB1 in 2, and KIT, EGFR, BRAF, STK11, ABL1, RB1 in one case each. Concomitant TP53 mutation was identified with other mutations like CDKN2A, HRAS, KIT, PIK3CA, STK11, SMARCB1, ABL1, and RB1 making tobacco-associated OSCC as a heterogeneous mutational tumor with multiple events. A patient with TP53 mutations has poor disease free survival (47.4 vs 63\% $p=0.17$ ); however, this was not statistically significant.

Conclusion: The study shows a heterogeneous mutational spectrum with multiple mutational events in OSCC. The low EGFR mutation rates and higher mutations in EGFR downstream pathways including that in TP53 and HRAS suggest that anti EGFR strategies may not succeed in these tumors and newer agents and therapeutic combinations need to be tried.

\section{Introduction}

Oral squamous cell cancer (OSCC) is the most common malignancy among males in India and the eighth most common cancer worldwide [1]. In India, gingivobuccal region of oral cavity is predominantly affected, comprising buccal mucosa and lower gums, whereas in the west, tongue is the most commonly involved subsite of oral cancer $[2,3]$. The risk factors for OSCC involve an interaction between the habits, environmental (tobacco, betel quid, alcohol, HPV, etc.), and genetic (EGFR, TP53, CDKN2A, etc.) factors [4-8].

EGFR is a member of receptor protein tyrosine kinase family with $42-80 \%$ over expression in head neck squamous cell carcinoma (HNSCC), whereas EGFR gene

\footnotetext{
* Correspondence: manojpandey66@gmail.com

Department of Surgical Oncology, Institute of Medical Sciences, Banaras Hindu University, Varanasi 221005, India
}

amplification is seen in up to $30 \%$ of HNSCC, and yet the results of EGFR targeting are not satisfactory. Since the approval of EGFR targeting drug, cetuximab for locally or regionally advanced and for metastatic HNSCC a lot of data has been generated on its use [9-12]. The EXTREME trial showed some treatment success with cetuximab plus platinum based chemotherapy in HNSCC; however, EGFR expression level was not found to be clinically useful predictive biomarker [13]. In platinum-refractory $\mathrm{HNSCC}$, the response rate with cetuximab monotherapy is only $10 \%$ [14]. SPECTRUM trial compared cisplatin/5-FU plus panitumumab to cisplatin/5-FU alone in patients with metastatic/recurrent SCCHN and showed significant activity of panitumumab [15]. Despite near universal expression of EGFR in HNSCC, there is only modest activity of these monoclonal antibodies. 
Other than anti-EGFR monoclonal antibodies (mAb), EGFR tyrosine kinase inhibitors (TKI) have also been tried with mixed success. Afatinib (selective EGFR and HER2 inhibitor), erlotinib (an oral reversible EGFR TKI), and gefitinib (a reversible EGFR TKI) have been used in cases with EGFR exon 19 deletions or exon 21 (L858R) substitution mutations as detected by an FDA-approved test. Vamurafenib (an oral selective inhibitor of BRAF kinase V600E oncogene) in unresectable and metastatic melanoma with the BRAFV600E mutation have been recently approved [11-16]. But still mono therapy with TKI's has only modest activity in EGFR mutated HNSCC [17].

Specific genetic mutations in HNSCC had been identified by next generation sequencing (NGS), some of which are potential targets and therapies can be tailored to augment existed EGFR targeted therapies [18]. The earlier results have shown mutation of the TP53, CDKN2A, HRAS, and PIK3CA genes [19-21] in downstream EGFR pathways, and hence can explain moderate activity of anti EGFR therapies in OSSC.

The Cancer Genome Atlas (TCGA) data profiling 279 HNSCCs showing mutations of the oncogene PIK3CA, novel alterations involving loss of TRAF3, and amplification of the cell-cycle gene E2F1 [22]. It also showed that tobacco-associated tumors showed high frequency of mutations in TP53 and CDKN2A [22]. Further a mutation-based signature affecting ten genes (HRAS, BRAF, FGFR3, SMAD4, KIT, PTEN, NOTCH1, AKT1, CTNNB1, and PTPN11) had been found to predict disease free survival (DFS) [19]. Despite identification of over 1500 mutation signatures in various genes in HNSCC, the data is still insufficient to plan therapeutic strategies based on these, and there is need to identify and establish potential genetic biomarkers and targets.

Further, personalized medicine, identification of optimal responders to targeted therapy, and creation of large databases integrating clinical and genetic data will help provide a panel of useful predictive biomarkers that will subsequently change clinical practices, and this study is a small step in this direction.

\section{Patients and methods}

This study was conducted in the Department of Surgical Oncology, Institute of Medical Sciences, Banaras Hindu University. Histologically proven cases of primary OSCC with history of tobacco intake were included, and patients with history of prior chemotherapy (for any reason) or radiotherapy were excluded. After the approval of the ethical committee and obtaining the consent, patients were recruited between 1st January 2017 and 1st January 2019.

Comprehensive history and physical examination was taken, and all the details were recorded in the preset pro forma. A biopsy to establish the diagnosis and CT scan of the head and neck to measure the tumor dimensions and stage the disease were performed before initiation of treatment. After the surgery the specimen was submitted for detailed histopathological examination. The archival tissue (formalin-fixed paraffin-embedded) was studied for expression of 50 genes by molecular analysis using next generation sequencing.

\section{Next generation sequencing DNA isolation/extraction}

DNA isolation from FFPE tissue with deparaffinization using ReliaPrep ${ }^{\text {ma }}$ FFPE gDNA Miniprep System, Promega Corporation, India, was carried out following the manufacturer's instruction protocol. For sections $\leq 50$ $\mu \mathrm{m}, 300 \mu \mathrm{l}$ of mineral oil and for sections $>50 \mu \mathrm{m}, 500$ $\mu \mathrm{l}$ of mineral oil was used for deparaffinization and incubate at $80{ }^{\circ} \mathrm{C}$ for $1 \mathrm{~min}$. Added $20 \mu \mathrm{l}$ of proteinase $\mathrm{K}$ directly to the lower phase and mixed by pipetting and incubate at $56{ }^{\circ} \mathrm{C}$ for $1 \mathrm{~h}$ and then at $80^{\circ} \mathrm{C}$ for $4 \mathrm{~h}$. The sample was allowed to cool at room temperature. $10 \mu \mathrm{l}$ of RNase was added to the lysed sample in the lower phase. The lower phase was mixed by pipetting and incubated at room temperature $\left(20-25{ }^{\circ} \mathrm{C}\right)$ for $5 \mathrm{~min}$.

\section{Template preparation}

Template preparation was done by building a library of nucleic acids (DNA or complementary DNA (cDNA) and amplifying that library. DNA Library preparation was carried out using an Ion PGM $^{\mathrm{m}}$ System, (Thermo Fisher Scientific, San Francisco, CA, USA) according to the manufacturer's instructions. Bar coded libraries using Ion Ampliseq Cancer Hotspot Panel v2 (Thermo Fisher Scientific) research panel were created. The Ion AmpliSeq $^{\mathrm{Tm}}$ Cancer Hotspot Panel v2 was designed to amplify 207 amplicons covering approximately 2800 COSMIC mutations from 50 oncogenes and tumor suppressor genes (ABL1, EGFR, GNAS, KRAS, PTPN11, AKT1, ERBB2, GNAQ, MET, RB1, ALK, ERBB4, HNF1A, MLH1, RET, APC, EZH2, HRAS, MPL, SMAD4, ATM, FBXW7, IDH1, NOTCH1, SMARCB1, BRAF, FGFR1, JAK2, NPM1, SMO, CDH1, FGFR2, JAK3, NRAS, SRC, CDKN2A, FGFR3, IDH2, PDGFRA, STK11, CSF1R, FLT3, KDR, PIK3CA, TP53, CTNNB1, GNA11, KIT, PTEN, VHL) with specific coverage of KRAS, HRAS, NRAS, BRAF, and EGFR exon 18-21with performance of at least $2000 \times$ sequence coverage for eight samples on one Ion 316 chip. In addition, the primers in this panel were designed to produce, on average, 154-bp amplicons, so that even degraded samples were used to generate reliable data. Bar coded libraries were combined to a final concentration of $100 \mathrm{pM}$. The next step was amplification of libraries which was done 
Table 1 Clinical and histopathological findings

\begin{tabular}{lll}
\hline Clinical findings & Frequency & Percent \\
\hline Site of tumor & 13 & \\
Tongue & 24 & 28.3 \\
Buccal Mucosa & 62.2 \\
Lower Alveolus & 1 & 13.0 \\
Upper Alveolus & 2 & 2.20 \\
Lip & & 4.30
\end{tabular}

Side

Right 18

Left

Midline

Crossing midline

Number lesion

single

Multiple

Clinical size of tumor $(\mathrm{cm})^{*}$

$$
\geq 4
$$

$<4$

Extension to adjacent site

$\begin{array}{ll}\text { Yes } & 17 \\ \text { No } & 29\end{array}$

T status

T2
T3
T4

Clinical nodes

No
N1
N3

Stage

॥

III

IVa

$\mathrm{IVb}$

Comorbid factors

Yes

No

ECOG performance status

$\begin{array}{ll}0 & 9 \\ 1 & 37\end{array}$

Imaging

$\mathrm{CT}$ size $(\mathrm{cm})$

$$
<4
$$$$
\geq 4
$$

33

17.40

32.60

37.00

28.30

2.20

37.00

37.00

2.20

17.40

82.60

19.60

80.40
Table 1 Clinical and histopathological findings (Continued)

\begin{tabular}{lll}
\hline Clinical findings & Frequency & Percent \\
\hline Neck node present & 22 & 47.80 \\
Involved adjacent site & & \\
$\quad$ Bone & 9 & 19.60 \\
$\quad$ Skin & 5 & 10.90 \\
$\quad$ Muscle & 9 & 19.60 \\
TNM stage (CT) & & \\
II & 11 & 23.90 \\
III & 17 & 37.00 \\
IVa & 17 & 37.00 \\
IVb & 1 & 2.00 \\
Neck dissection & & \\
SOHND & 22 & 47.80 \\
MRND & 17 & 37.00 \\
None & 7 & 15.20 \\
Surgical reconstruction & & \\
None & 16 & 34.80 \\
Pectoralis major myocutaneous flap & 14 & 30.40 \\
Nasolabial flap & 13 & 28.30 \\
Buccal pad of fat & 2 & 4.30 \\
Sternocleidomastoid flap & 1 & \\
Total & 46 &
\end{tabular}

$50.00 \quad$ Histopathological findings

HPE grade

I (well differentiated)

22

47.80

II (moderately differentiated)

47.80

III (poorly differentiated)

4.30

T size $(\mathrm{cm})$

$\geq 4$

23.90

$<4$

76.10

Positive margin

4.30

Lymphovascular invasion

28.30

Perineural invasion

43.50

Depth of invasion ( $\mathrm{mm}$ )

$<5$

8.70

$\geq 5$ to $<10$

52.20

$\geq 10$

39.10

Pathological stage

।

2.20

॥

47.80

III

IVa

$\mathrm{IVb}$

15.20

28.30

Mutations

$\begin{array}{lr}28.30 & \text { Mutations } \\ 71.70 & \text { TP53 }\end{array}$
6.50

41.3 
Table 1 Clinical and histopathological findings (Continued)

\begin{tabular}{lll}
\hline Clinical findings & Frequency & Percent \\
\hline CDKN2A & 11 & 23.9 \\
HRAS & 8 & 17.4 \\
PIK3CA & 3 & 6.5 \\
SMARCB1 & 2 & 4.3 \\
KIT & 1 & 2.2 \\
EGFR & 1 & 2.2 \\
BRAF & 1 & 2.2 \\
STK11 & 1 & 2.2 \\
ABL1 & 1 & 2.2 \\
RB1 & 1 & 2.2 \\
NRAS & 0 & 0.0 \\
KRAS & 0 & 0.0 \\
\hline
\end{tabular}

*The largest numeric value of length, breadth, and depth had been considered

by using emulsion PCR (emPCR) on the 2 Ion OneTouch system (Life Technologies).

\section{Sequencing and imaging}

The Ion PGM ${ }^{\mathrm{TM}}$ Torrent relied on the library fragments which acted as a template, off which a new DNA fragment was synthesized. As nucleotides were incorporated into the growing DNA strand, they were digitally recorded as sequence. Sequencing primer and polymerase were added to the final enriched ISPs prior to loading onto 316 (100 Mb output) chips. Alignment of sequences was performed using Torrent Suite ${ }^{\mathrm{TM}}$ Software (version5.2.0) on the Ion PGM $^{\mathrm{TM}}$ Torrent Server.

\section{Sequencing data analysis}

After sequencing, preprocessing the data to remove adapter sequences and low-quality reads was carried out followed by mapping of the data to a reference genome or de novo alignment of the sequence reads, and analysis of the compiled sequence using wide variety of bioinformatics assessments, including genetic variant calling for detection of SNPs or indels (i.e., the insertion or deletion of bases), detection of novel genes or regulatory elements, and assessment of transcript expression levels. Data analysis was carried out with Torrent Suite Software V.5.2.0 (Life Technologies). The Ion Reporter suite (Life Technologies) was used to filter polymorphic variants.

\section{Statistical analysis}

Statistical analysis was performed using SPSS version 23.0 (IBM Corp., Armonk, NY). The baseline characteristics were compared using the chi-square and Fisher Exact test. Survival was estimated using Kaplan-Meier method and was compared using log-rank test. Statistical significance was set at $P<0.05$.

\section{Results}

The mean age of patients was $47.8 \pm 10.9$ years. Majority of cases were male (93.5\%) followed by females with male: female ratio of 14.3:1. Only 1 patient had family history of cancer. Leukoplakia was present in 2 (4.3\%) cases, and others had no precancerous lesion. The clinical and histopathological findings and treatment is presented in Table 1.

Out of 46 cases, 19 (41.3\%) had received adjuvant radiotherapy, and $8(17.4 \%)$ had received adjuvant chemotherapy. There were total 20 recurrences, 17 recurrences occurred at primary site, 2 were the lymph node recurrences, and 1 was the second primary. All stage III, IVa, and IVb were advised adjuvant radiotherapy, but 6 cases did not take, of these 2 cases had recurrence at primary site which could not be re-excised and were started on palliative chemotherapy. Out of 19 cases who were given adjuvant radiotherapy in 9 had locoregional recurrence.

TP53 was the most common (41.3\%) mutation followed by CDKN2A (23.9\%), HRAS (17.4\%), PIK3CA (6.5\%), SMARCB1 (4.3\%), and KIT, EGFR, BRAF, STK11, ABL1, RB1 (2.2\% each). The relationship of TP53, CDKN2A, PIK3CA, and HRAS mutation with clinical-pathological factors are presented in Table 2. The HRAS and PIK3CA mutation had significant association with site of tumor, i.e., lower lip $(p=0.002)$ and lower alveolus $(p=0.004)$.

The median follow-up in this study is 19.3 months, and the median disease free survival was 16 months. The 30 months OS rates and DFS rates of TP53, CDKN2A, PIK3CA, and HRAS is depicted in Table 3, the details of the identified mutation are recorded in Table 4 . The disease free survival rates were $47.4 \%$ and $63 \%$ for TP53 mutation present and absent respectively (Log rank = 1.44; $p=0.17)$ (Fig. 1).

\section{Discussion}

Incidence of OSCC has increased with widespread use of smokeless tobacco, betel quid, HPV infection, environmental pollution, and genetic risk factors. Anatomically, the oral cavity is composed of the mucosal lip, oral tongue, floor of mouth, mandibular and maxillary gingiva, retromolar trigone, buccal mucosa, and hard palate subsites controlling the speech, swallowing, and facial projections. Surgery is the mainstay treatment for OSCC, despite current advances in the treatment options, the fatality of oral cancer has remained mostly unchanged. In addition to radiotherapy, anti-EGFR strategies have shown improved outcomes in the adjuvant setting and have become an active area of research. 
Table 2 Relationship of TP53, CDKN2A, PIK3CA, HRAS mutation with clinical-pathological factors

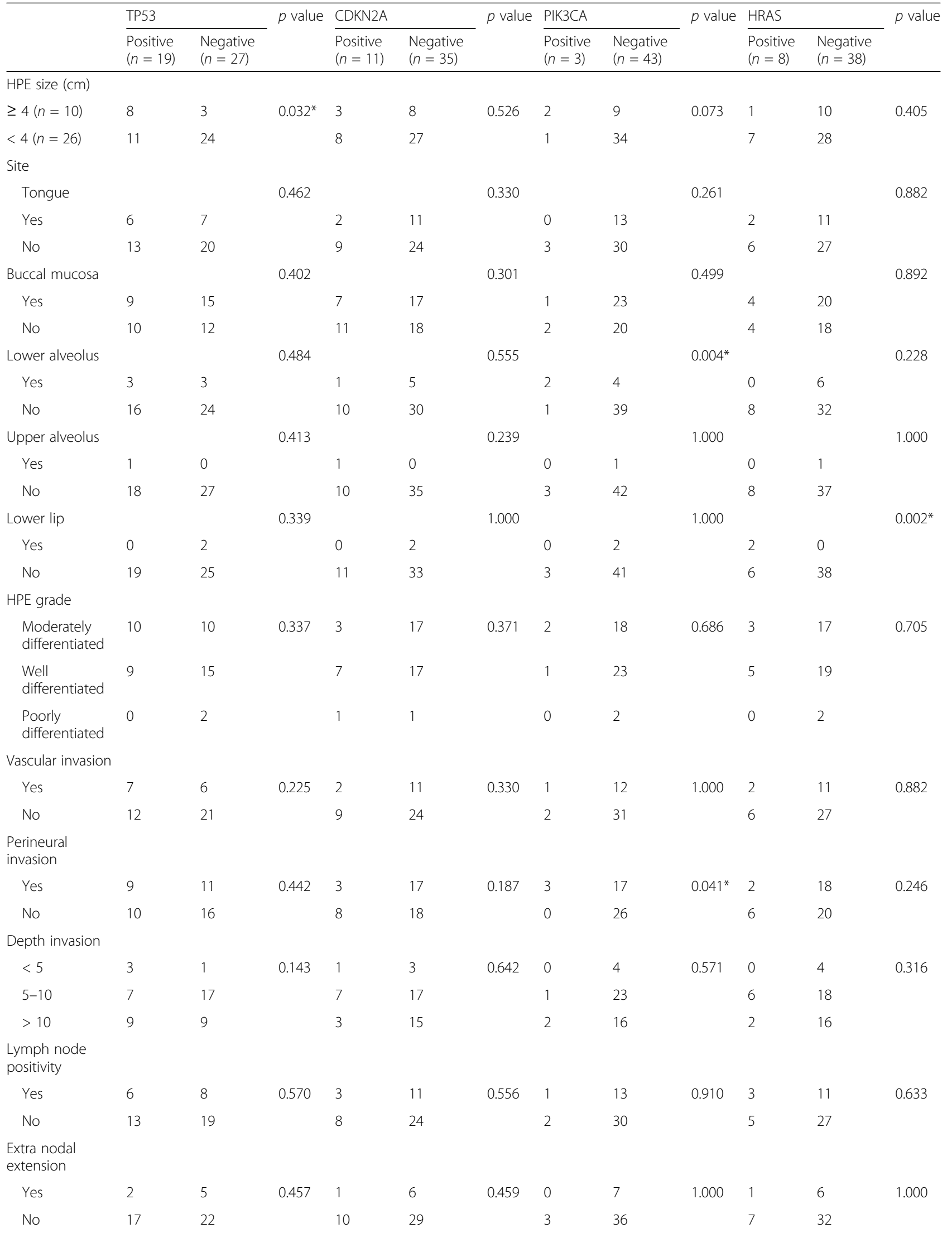


Table 2 Relationship of TP53, CDKN2A, PIK3CA, HRAS mutation with clinical-pathological factors (Continued)

\begin{tabular}{|c|c|c|c|c|c|c|c|c|c|c|c|c|}
\hline & \multicolumn{2}{|l|}{ TP53 } & \multirow[t]{2}{*}{$p$ value } & \multicolumn{2}{|l|}{ CDKN2A } & \multirow[t]{2}{*}{$p$ value } & \multicolumn{2}{|l|}{ PIK3CA } & \multirow[t]{2}{*}{$p$ value } & \multicolumn{2}{|l|}{ HRAS } & \multirow[t]{2}{*}{$p$ value } \\
\hline & $\begin{array}{l}\text { Positive } \\
(n=19)\end{array}$ & $\begin{array}{l}\text { Negative } \\
(n=27)\end{array}$ & & $\begin{array}{l}\text { Positive } \\
(n=11)\end{array}$ & $\begin{array}{l}\text { Negative } \\
(n=35)\end{array}$ & & $\begin{array}{l}\text { Positive } \\
(n=3)\end{array}$ & $\begin{array}{l}\text { Negative } \\
(n=43)\end{array}$ & & $\begin{array}{l}\text { Positive } \\
(n=8)\end{array}$ & $\begin{array}{l}\text { Negative } \\
(n=38)\end{array}$ & \\
\hline \multicolumn{13}{|c|}{ T status } \\
\hline $\mathrm{T} 2$ & 8 & 15 & 0.660 & 8 & 15 & 0.127 & 0 & 23 & $0.036^{*}$ & 5 & 18 & 0.057 \\
\hline T3 & 4 & 4 & & 0 & 8 & & 0 & 8 & & 3 & 5 & \\
\hline T4 & 7 & 8 & & 3 & 12 & & 3 & 12 & & 0 & 15 & \\
\hline \multicolumn{13}{|l|}{ Stage } \\
\hline 1 & 1 & 0 & 0.453 & 0 & 1 & 0.282 & 0 & 1 & 0.086 & 0 & 1 & 0.880 \\
\hline$\|$ & 9 & 13 & & 8 & 14 & & 0 & 22 & & 4 & 18 & \\
\hline III & 3 & 4 & & 0 & 7 & & 0 & 7 & & 1 & 6 & \\
\hline IVa & 6 & 7 & & 2 & 11 & & 3 & 10 & & 3 & 10 & \\
\hline $\mathrm{IVb}$ & 0 & 3 & & 1 & 2 & & 0 & 3 & & 0 & 3 & \\
\hline
\end{tabular}

*statistically significant

The use of next generation sequencing (NGS) in HNSCC has led to identification of novel mutated tumor suppressor genes (TP53, CDKN2A) and oncogenes (PIK3CA, HRAS, EGFR) and has led to the development of predictive biomarkers. There are several genetic alterations responsible for development of OSCC; out of which, EGFR is a validated target, other than PI3K, PTEN, VEGF, JAK-STAT, etc. However, in presence of downstream mutations the EGFR targeting is mostly ineffective.

In our study, concomitant TP53 mutation was present with other mutations like CDKN2A, HRAS, KIT, PIK3CA, STK11, SMARCB1, ABL1, and RB1 making oral cavity squamous cell cancer as a heterogeneous mutational tumor. There were 16 different TP53 mutations in exons 4-8, out which most commonly was found on codon 72 , c. $215 \mathrm{C}>\mathrm{G}$ (Pro72Arg) transversion variant in 5 patients (Table 4). This variant had been demonstrated in oral cavity squamous cell cancers (OSCC) previously [23]. Similarly codon 248 c.743G $>$ A (Arg248Gln) and codon 306 c.916C $>\mathrm{T}$ (Arg306Ter) had been reported earlier [24]. An intron splice variant c.376-1G>A [25] and mutations in codon 152 [26], 158 [27], 165 [28], 204 [29], and 241 [30] were also previously reported in OSCC. Whereas codon 244 c.731G $>\mathrm{C}$ (Gly244Ala) and codon c.799C $>\mathrm{T}$ (Arg267Tryp) had never been reported in OSCC but has been reported in other cancers [31, 32]. Mutation on codon 274 c.821T>G (Val274Gly) and a deletion frameshift variant at codon 301 (Pro301Glnfs) had also never been reported in OSCC or in other cancers and were a novel finding in this study.

EGFR point mutations are reported in $9 \%$ patients in a study conducted by Dubot et al. in 2017 [33]; however, we have identified only one EGFR mutation on exon 19, codon 746 (Glu746del) which is an in-frame deletion mutation. This mutation has been previously reported by Ragga et al. in 2006 in HNSCC [34].

The point mutations that activate RAS protooncogenes have been found to be located mainly at the codons 12, 13, and 61 [35] and is estimated to be $15 \%$ [36]. In our study, the HRAS found to be mutated in $17.4 \%$ patients. All these mutations are previously reported in OSCC and have high incidence in lip vermilion cancers [37-39].

V600E mutation is the most frequently identified cancer-causing mutations in melanoma and other malignancies like non-Hodgkin lymphoma, colorectal cancer, thyroid carcinoma, non-small cell lung carcinoma, hairy cell leukemia, and adenocarcinoma of lung. In our study, we found a transversion mutation in BRAF exon11 codon 466 c.1397G >C (Gly466Ala), in a patient of carcinoma lower alveolus. This mutation has been reported in COSMIC database and CLINVAR database within exons 11 and 15 with a predominant nucleotide change at codons 599 and 468 in HNSCC (pharynx) [40]. Another study reported BRAF mutation in exon15, codon 412 in maxillary alveolus [41]. After having a thorough search of genetic mutation databases, we found that the mutation in BRAF exon 11 c.1397G $>C$ (Gly466Ala) has never been reported in lower alveolus.

Of the 11, CDKN2A mutations identified in this study, 10 were non-sense mutations, and one was frameshift deletion. The most common mutation which was present in 6 patients, i.e., c. $238 \mathrm{C}>\mathrm{T}$ (Arg80Ter) was found to be frequently reported mutation in OSCC. Similarly, c.330G $>$ A (Trp110Ter) present in three patients was also found to be a known mutation in oral cavity SCC [42]. Further, we found a novel mutation on codon 78, c.233-234delTC (Leu78fs) in a patient of carcinoma buccal mucosa. 
Table 3 Disease free survival and overall survival of various clinicopathological factors and studied mutations

\begin{tabular}{|c|c|c|c|c|}
\hline Variables & 30 month disease free survival (\%) & $p$ value & 30 month overall survival (\%) & $p$ value \\
\hline Age (year) & & $0.001^{*}$ & & $0.003^{*}$ \\
\hline$\leq 46$ & 36.4 & & 45.5 & \\
\hline$>46$ & 75.0 & & 87.5 & \\
\hline Site of tumor & & 0.641 & & 0.665 \\
\hline Tongue & 61.5 & & 69.2 & \\
\hline Buccal mucosa & 50.0 & & 66.7 & \\
\hline Lower alveolus & 66.7 & & 50.0 & \\
\hline Upper alveolus & 100 & & 100 & \\
\hline Lower lip & 50.0 & & 100 & \\
\hline HPE size $(\mathrm{cm})$ & & 0.846 & & 0.720 \\
\hline$<4$ & 54.3 & & 74.3 & \\
\hline$\geq 4$ & 63.6 & & 45.5 & \\
\hline Lymph node (HPE) & & 0.250 & & $0.021^{*}$ \\
\hline Positive & 42.9 & & 42.9 & \\
\hline Negative & 62.5 & & 78.1 & \\
\hline Extra nodal extension (ENE) & & 0.464 & & 0.997 \\
\hline Present & 42.9 & & 71.4 & \\
\hline Absent & 59.0 & & 66.7 & \\
\hline Depth of invasion (mm) & & 0.731 & & $0.008^{*}$ \\
\hline$<5$ & 50.0 & & 100 & \\
\hline$\geq 5,>10$ & 58.3 & & 79.2 & \\
\hline$<10$ & 55.6 & & 44.4 & \\
\hline Vascular invasion & & $0.018^{*}$ & & $0.042^{*}$ \\
\hline Positive & 23.1 & & 38.5 & \\
\hline Negative & 69.7 & & 78.8 & \\
\hline $\mathrm{PNI}$ & & 0.196 & & 0.134 \\
\hline Positive & 40.0 & & 50.0 & \\
\hline Negative & 69.2 & & 80.8 & \\
\hline Adjuvant RT & & 0.731 & & $0.042^{*}$ \\
\hline Yes & 52.6 & & 47.4 & \\
\hline No & 59.3 & & 81.5 & \\
\hline \multicolumn{5}{|l|}{ Mutations } \\
\hline TP53 & & 0.176 & & 0.082 \\
\hline Present & 47.4 & & 52.6 & \\
\hline Absent & 63.0 & & 77.8 & \\
\hline CDKN2A & & 0.320 & & 0.431 \\
\hline Present & 72.7 & & 81.8 & \\
\hline Absent & 51.4 & & 62.9 & \\
\hline PIK3CA & & 0.148 & & 0.221 \\
\hline Present & 100 & & 100 & \\
\hline Absent & 53.5 & & 65.1 & \\
\hline HRAS & & 0.629 & & 0.761 \\
\hline Present & 62.5 & & 75.0 & \\
\hline Absent & 55.3 & & 65.8 & \\
\hline
\end{tabular}

*statistically significant 
Table 4 Mutational analysis of all study subjects

\begin{tabular}{|c|c|c|c|c|c|c|}
\hline S. No. & Mutations & Cytogenetic location & Codon no. & Variant & Variant type & Site \\
\hline 1 & TP53 & $17 p 13.1$ & Codon 72 & $\begin{array}{l}\text { c.215C>G } \\
\text { (Pro72Arg) }\end{array}$ & $\begin{array}{l}\text { Mis-sense } \\
\text { Transversion }\end{array}$ & $\overline{\text { Buccal mucosa }}$ \\
\hline 2 & TP53 & 17p13.1 & Codon 72 & $\begin{array}{l}\text { c.215C>G } \\
\text { (Pro72Arg) }\end{array}$ & $\begin{array}{l}\text { Mis-sense } \\
\text { Transversion }\end{array}$ & Buccal mucosa \\
\hline 3 & TP53 & $17 p 13.1$ & Codon 72 & $\begin{array}{l}\text { c.215C>G } \\
\text { (Pro72Arg) }\end{array}$ & $\begin{array}{l}\text { Mis-sense } \\
\text { Transversion }\end{array}$ & Tongue \\
\hline 4 & TP53 & 17p13.1 & Codon 72 & $\begin{array}{l}\text { c. } 215 C>G \\
\text { (Pro72Arg) }\end{array}$ & $\begin{array}{l}\text { Mis-sense } \\
\text { Transversion }\end{array}$ & Tongue \\
\hline 5 & TP53 & 17p13.1 & Codon 72 & $\begin{array}{l}\text { c.215C>G } \\
\text { (Pro72Arg) }\end{array}$ & $\begin{array}{l}\text { Mis-sense } \\
\text { Transversion }\end{array}$ & Buccal mucosa \\
\hline 6 & TP53 & 17p13.1 & Codon 152 & $\begin{array}{l}\text { c.455C>T } \\
\text { (Pro152Leu) }\end{array}$ & $\begin{array}{l}\text { Mis-sense } \\
\text { Transition }\end{array}$ & Lower alveolus \\
\hline 7 & TP53 & 17p13.1 & Codon 158 & $\begin{array}{l}\text { c.472C>T } \\
\text { (Arg158Cys) }\end{array}$ & $\begin{array}{l}\text { Mis-sense } \\
\text { Transition }\end{array}$ & Tongue \\
\hline 8 & TP53 & $17 p 13.1$ & Codon 165 & $\begin{array}{l}\text { c.493C>T } \\
\text { (Gln165Ter) }\end{array}$ & Non-sense & Tongue \\
\hline 9 & TP53 & $17 p 13.1$ & Codon 173 & $\begin{array}{l}\text { C.517G }>\text { A } \\
\text { (Val173Met) }\end{array}$ & $\begin{array}{l}\text { Mis-sense } \\
\text { Transition }\end{array}$ & Tongue \\
\hline 10 & TP53 & 17p13.1 & Codon 175 & $\begin{array}{l}\text { C.524G>A } \\
\text { (Arg175His) }\end{array}$ & $\begin{array}{l}\text { Mis-sense } \\
\text { Transition }\end{array}$ & Buccal mucosa \\
\hline 11 & TP53 & 17p13.1 & Codon 204 & $\begin{array}{l}\text { C.610G >T } \\
\text { (Glu204Ter) }\end{array}$ & Non-sense & Buccal mucosa \\
\hline 12 & TP53 & 17p13.1 & Codon 213 & $\begin{array}{l}\text { c.638G>A } \\
(\text { Arg213GIn) }\end{array}$ & $\begin{array}{l}\text { Mis-sense } \\
\text { Transition }\end{array}$ & Buccal mucosa \\
\hline 13 & TP53 & 17p13.1 & Codon 241 & $\begin{array}{l}\text { c.722C>T } \\
\text { (Ser241Phe) }\end{array}$ & $\begin{array}{l}\text { Mis-sense } \\
\text { Transition }\end{array}$ & Lower alveolus \\
\hline 14 & TP53 & $17 p 13.1$ & Codon 244 & $\begin{array}{l}\text { c.731G>C } \\
\text { (Gly244Ala) }\end{array}$ & $\begin{array}{l}\text { Mis-sense } \\
\text { Transversion }\end{array}$ & Buccal mucosa \\
\hline 15 & TP53 & $17 p 13.1$ & Codon 248 & $\begin{array}{l}\text { c.742C>T } \\
\text { (Arg248Trp) }\end{array}$ & $\begin{array}{l}\text { Mis-sense } \\
\text { Transition }\end{array}$ & Buccal mucosa \\
\hline 16 & TP53 & 17p13.1 & Codon 248 & $\begin{array}{l}\text { c.742C>T } \\
\text { (Arg248Trp) }\end{array}$ & $\begin{array}{l}\text { Mis-sense } \\
\text { Transition }\end{array}$ & Tongue \\
\hline 17 & TP53 & 17p13.1 & Codon 248 & $\begin{array}{l}\text { c.743G }>A \\
(\operatorname{Arg} 248 G \ln )\end{array}$ & $\begin{array}{l}\text { Mis-sense } \\
\text { Transition }\end{array}$ & Buccal mucosa \\
\hline 18 & TP53 & 17p13.1 & Codon 267 & $\begin{array}{l}\text { c.799C>T } \\
\text { (Arg267Tryp) }\end{array}$ & $\begin{array}{l}\text { Mis-sense } \\
\text { Transition }\end{array}$ & Tongue \\
\hline 19 & TP53 & 17p13.1 & Codon 274 & $\begin{array}{l}\text { C.820G>T } \\
\text { (Val274Phe) }\end{array}$ & $\begin{array}{l}\text { Mis-sense } \\
\text { Tranversion }\end{array}$ & Lower alveolus \\
\hline 20 & TP53 & $17 p 13.1$ & Codon 274 & $\begin{array}{l}\text { c.821T>G } \\
\text { (Val274Gly) }\end{array}$ & $\begin{array}{l}\text { Mis-sense } \\
\text { Transversion }\end{array}$ & Buccal mucosa \\
\hline 21 & TP53 & 17p13.1 & Codon 301 & c.902delC (pro301Glnfs) & Deletion (frameshift) & Tongue \\
\hline 22 & TP53 & 17p13.1 & Codon 306 & $\begin{array}{l}\text { c.916C>T } \\
\text { (Arg306Ter) }\end{array}$ & Non-sense & Buccal mucosa \\
\hline 23 & TP53 & 17p13.1 & Codon 306 & $\begin{array}{l}\text { c.916C>T } \\
\text { (Arg306Ter) }\end{array}$ & Non-sense & Upper alveolus \\
\hline 24 & TP53 & $17 p 13.1$ & - & $\begin{array}{l}\text { c.376-1G>A } \\
\text { unknown }\end{array}$ & $\begin{array}{l}\text { Intron variant } \\
\text { (splice site) }\end{array}$ & Buccal mucosa \\
\hline 25 & CDKN2A & $9 p 21.3$ & Codon78 & $\begin{array}{l}\text { c.233-234delTC } \\
\text { (Leu78fs) }\end{array}$ & $\begin{array}{l}\text { Deletion } \\
\text { (frameshift) }\end{array}$ & Buccal mucosa \\
\hline 26 & CDKN2A & $9 p 21.3$ & Codon 80 & $\begin{array}{l}\text { c. } 238 \text { C>T } \\
\text { (Arg80Ter) }\end{array}$ & Non-sense & Buccal mucosa \\
\hline 27 & CDKN2A & $9 p 21.3$ & Codon 80 & $\begin{array}{l}\text { C.238 C>T } \\
\text { (Arg80Ter) }\end{array}$ & Non-sense & Buccal mucosa \\
\hline
\end{tabular}


Table 4 Mutational analysis of all study subjects (Continued)

\begin{tabular}{|c|c|c|c|c|c|c|}
\hline S. No. & Mutations & Cytogenetic location & Codon no. & Variant & Variant type & Site \\
\hline$\overline{28}$ & CDKN2A & $9 p 21.3$ & Codon 80 & $\begin{array}{l}c .238 C>\top \\
\text { (Arg80Ter) }\end{array}$ & Non-sense & Buccal mucosa \\
\hline 29 & CDKN2A & $9 p 21.3$ & Codon 80 & $\begin{array}{l}\text { c.238C }>\text { T } \\
\text { (Arg80Ter) }\end{array}$ & Non-sense & Tongue \\
\hline 30 & CDKN2A & $9 p 21.3$ & Codon 80 & $\begin{array}{l}\text { c.238C }>\text { T } \\
\text { (Arg80Ter) }\end{array}$ & Non-sense & Lower alveolus \\
\hline 31 & CDKN2A & $9 p 21.3$ & Codon 80 & $\begin{array}{l}\text { c.238C }>T \\
\text { (Arg80Ter) }\end{array}$ & Non-sense & Tongue \\
\hline 32 & CDKN2A & $9 p 21.3$ & Codon 110 & $\begin{array}{l}\text { c.330G>A } \\
\text { (Trp110Ter) }\end{array}$ & Non-sense & Buccal mucosa \\
\hline 33 & CDKN2A & $9 p 21.3$ & Codon 110 & $\begin{array}{l}\text { c.330G>A } \\
\text { (Trp110Ter) }\end{array}$ & Non-sense & Buccal mucosa \\
\hline 34 & CDKN2A & $9 p 21.3$ & Codon 110 & $\begin{array}{l}\text { C.330G>A } \\
\text { (Trp110Ter) }\end{array}$ & Non-sense & Upper alveolus \\
\hline 35 & HRAS & $11 \mathrm{p} 15.5$ & Codon 12 & $\begin{array}{l}\text { c.34G>A } \\
\text { (Gly12Ser) }\end{array}$ & $\begin{array}{l}\text { Mis-sense } \\
\text { Transition }\end{array}$ & Buccal mucosa \\
\hline 36 & HRAS & $11 p 15.5$ & Codon 12 & $\begin{array}{l}\text { c.34G>A } \\
\text { (Gly12Ser) }\end{array}$ & $\begin{array}{l}\text { Mis-sense } \\
\text { Transition }\end{array}$ & Buccal mucosa \\
\hline 37 & HRAS & $11 p 15.5$ & Codon 12 & $\begin{array}{l}\text { c.34G>A } \\
\text { (Gly12Ser) }\end{array}$ & $\begin{array}{l}\text { Mis-sense } \\
\text { Transition }\end{array}$ & Buccal mucosa \\
\hline 38 & HRAS & $11 p 15.5$ & Codon 12 & $\begin{array}{l}\text { c.35G }>A \\
\text { (Gly12Asp) }\end{array}$ & $\begin{array}{l}\text { Mis-sense } \\
\text { Transition }\end{array}$ & Lower lip \\
\hline 39 & HRAS & $11 p 15.5$ & Codon 13 & $\begin{array}{l}\text { c.37G }>C \\
\text { (Gly13Arg) }\end{array}$ & $\begin{array}{l}\text { Mis-sense } \\
\text { Transversion }\end{array}$ & Lower lip \\
\hline 40 & HRAS & $11 p 15.5$ & Codon 13 & $\begin{array}{l}\text { c.38G >T } \\
\text { (Gly13Val) }\end{array}$ & $\begin{array}{l}\text { Mis-sense } \\
\text { Transversion }\end{array}$ & Buccal mucosa \\
\hline 41 & HRAS & $11 p 15.5$ & Codon 61 & $\begin{array}{l}\text { C. } 181 C>A \\
\text { (Gln61Lys) }\end{array}$ & $\begin{array}{l}\text { Mis-sense } \\
\text { Transversion }\end{array}$ & Tongue \\
\hline 42 & HRAS & $11 p 15.5$ & Codon 61 & $\begin{array}{l}\text { c.182A>T } \\
\text { (Gln61Leu) }\end{array}$ & $\begin{array}{l}\text { Mis-sense } \\
\text { Transversion }\end{array}$ & Tongue \\
\hline 43 & PIK3CA & $3 q 26.32$ & Codon 542 & $\begin{array}{l}\text { C.1625A>C } \\
\text { (Glu542Ala) }\end{array}$ & $\begin{array}{l}\text { Mis-sense } \\
\text { Transversion }\end{array}$ & Lower alveolus \\
\hline 44 & PIK3CA & $3 q 26.3$ & Codon 542 & $\begin{array}{l}\text { c.1624G>A } \\
\text { (Glu542Lys) }\end{array}$ & $\begin{array}{l}\text { Mis-sense } \\
\text { Transition }\end{array}$ & Lower alveolus \\
\hline 45 & PIK3CA & $3 q 26.3$ & Codon 1047 & $\begin{array}{l}\text { c.3140 A>G } \\
\text { (His1047Arg) }\end{array}$ & $\begin{array}{l}\text { Mis-sense } \\
\text { Transition }\end{array}$ & Buccal mucosa \\
\hline 46 & SMARCB1 & $22 q 11.23$ & - & $\begin{array}{l}\text { C.1146-41G>A } \\
\text { unknown }\end{array}$ & Intron variant & Buccal mucosa \\
\hline 47 & SMARCB1 & $22 q 11.23$ & - & c. $1146-41 \mathrm{G}>\mathrm{A}$ & Intron variant & Buccal mucosa \\
\hline 48 & KIT & $4 q 12$ & Codon 541 & $\begin{array}{l}\text { C.1621A>C } \\
\text { (Met541Leu) }\end{array}$ & $\begin{array}{l}\text { Mis-sense } \\
\text { Transversion }\end{array}$ & Buccal mucosa \\
\hline 49 & BRAF & $7 q 34$ & Codon 466 & $\begin{array}{l}\text { c.1397G>C } \\
\text { (Gly466Ala) }\end{array}$ & $\begin{array}{l}\text { Mis-sense } \\
\text { Transversion }\end{array}$ & Lower alveolus \\
\hline 50 & STK11 & $19 p 13.3$ & Codon 357 & $\begin{array}{l}\text { c.1071G>T } \\
\text { (Glu357Asp) }\end{array}$ & $\begin{array}{l}\text { Mis-sense } \\
\text { Transversion }\end{array}$ & Lower alveolus \\
\hline \multirow[t]{2}{*}{51} & \multirow[t]{2}{*}{ ABL1 } & \multirow[t]{2}{*}{$9 q 34.12$} & Codon 274 & $\begin{array}{l}\text { c.764A>T } \\
\text { (Glu274Val) }\end{array}$ & $\begin{array}{l}\text { Mis-sense } \\
\text { Transversion }\end{array}$ & \multirow[t]{2}{*}{ Tongue } \\
\hline & & & Codon 415 & $\begin{array}{l}\text { C.1187A>G } \\
\text { (His415Pro) }\end{array}$ & $\begin{array}{l}\text { Mis-sense } \\
\text { Transition }\end{array}$ & \\
\hline 52 & EGFR & $7 p 11.2$ & Codon 746 & c.2234delAGG (Glu746del) & $\begin{array}{l}\text { Deletion } \\
\text { (in frame } \\
\text { Deletion) }\end{array}$ & Tongue \\
\hline 53 & RB1 & $13 q 14.2$ & Codon 680 & $\begin{array}{l}\text { c.2039T>C } \\
\text { (Ile680Thr) }\end{array}$ & $\begin{array}{l}\text { Mis-sense } \\
\text { Transition }\end{array}$ & Tongue \\
\hline
\end{tabular}




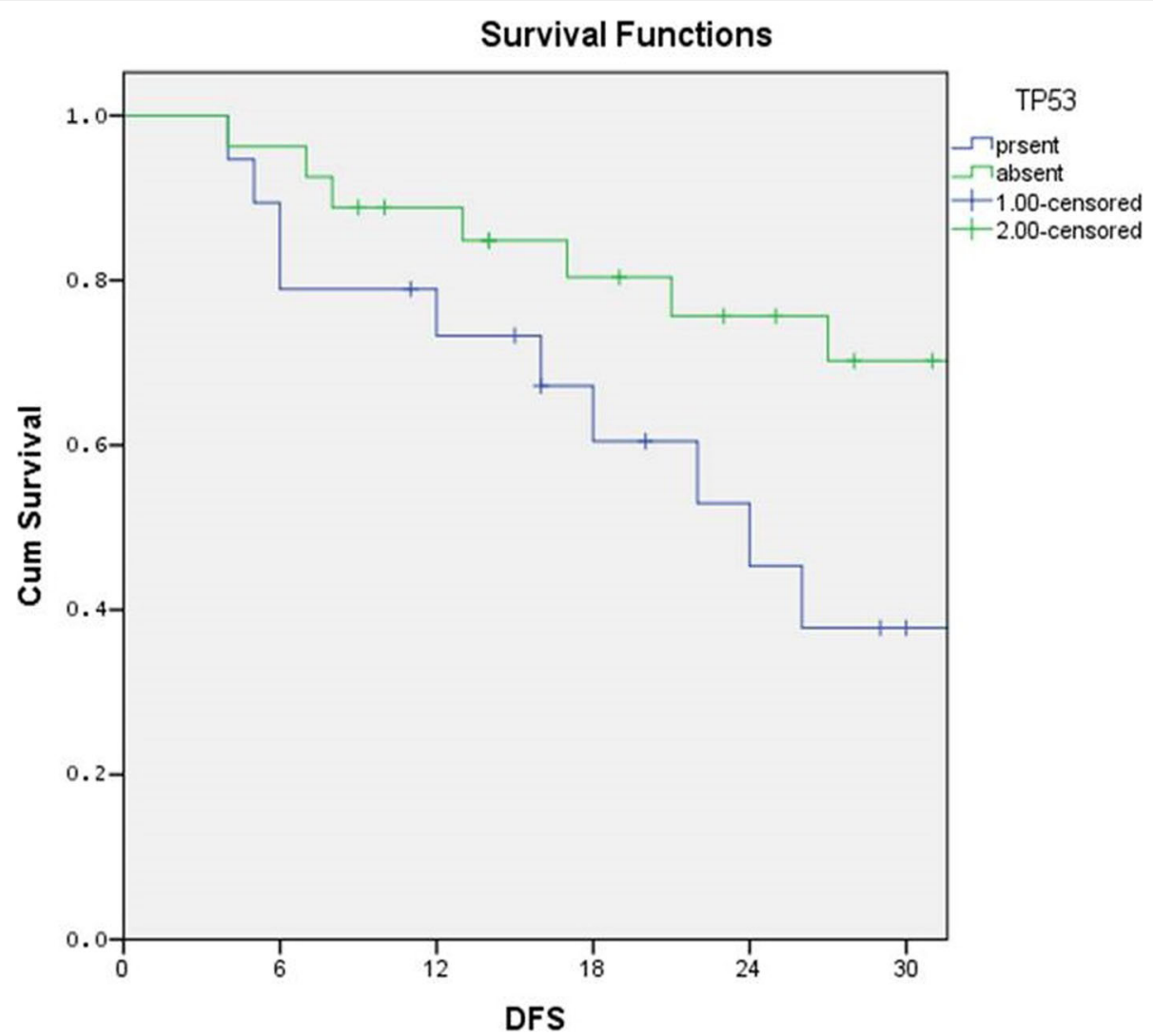

Fig. 1 Kaplan-Meier survival curve showing difference in survival between p53 mutated and wild groups. The 18 months DFS rates were 53.3\% and $71.4 \%$ for TP53 mutation present and absent respectively (log rank $=1.44 ; p=0.229$ )

The frequency of mutations of the PIK3CA gene has been reported in $11 \%$ HNSCC [43]. Kozaki et al. did mutational analysis of cell lines and primary tumors of OSCC, found a significant correlation between the advanced stage of OSCC and the frequency with which PIK3CA is mutated in exons 9 and 20 [44]. PIK3CA mutation was the most common mutation in HPV positive HNSCC while phosphate and tensin homolog (PTEN) loss were frequent event independent of HPV status $[45,46]$. PIK3CA is reported to be mutated in $12 \%-16 \%$ of HNSCC exome/genome analysis. In our study, percentage of PIK3CA mutation was $6.5 \%$ and was significantly associated with lower alveolus lesions. The codon variant His1047Arg and Glu542Lys have been reported in many studies as hotspot mutation sites in HNSCC, but Glu542Ala is only reported in cancers of the breast, endometrium, prostate and esophagus [47] and is for the first time being reported in OSCC.

KIT gene mutations have earlier been reported in gastrointestinal stromal tumors, chronic myeloid leukemia, etc.[48],but has never been reported in OSCC; however, it was found in one of our patients at exon 10, codon 541 c.1621A $>C$ (Met541Leu) as transversion mutation.
STK11 is a tumor-suppressor gene involved in causing Peutz Jeghers syndrome, but the role of STK11/LKB1 gene inactivation in neoplasia has not been conclusively demonstrated so far. Tan et al. conducted a study in 2014 on carcinoma tongue patients, compared their genetic mutations with Lung Carta 1.0 gene panel and found 9\% patients had STK11 mutations [49]. In our study, only one patient had STK11 mutation exon 8 codon 357 c.1071G $>$ T (Glu357Asp) transversion variant type that is different from those reported by Tan et al. and has never been reported before in OSCC.

SMARCB1 mutation c.1146-41G>A as intron variants were present in two patients which were also having Tp53 mutation along with, these mutations are not known to play any major role in tumorigenesis. Two ABL1 mutations were present in one patient of carcinoma tongue along with RB and TP53 mutations. ABL1 c.764A $>$ T (Glu274Val) and c.1187A $>$ G (His415Pro) mutations are commonly seen in patients of chronic myeloid leukemia and have never been reported in oral cavity squamous cell cancers. RB mutation is only reported in cases of carcinoma breast and thyroid, but we found a novel mutation c.2039T $>C$ (Ile680Thr) in one of our patients. 
The results of the present study suggest that the mutation spectrum of OSCC may be different in different races, with Indian OSCC showing some distinct mutations that has not been seen in Chinese and Caucasians reported earlier. It also show that the mutations vary by subsite within the oral cavity, though TP53, CDKN2A, and PIK3CA mutations could be the common event in all oral cavity subsites [50-52]. Despite the limitations of the sample size this study shows that mutations in tobacco associated cancers are high, and concomitant multiple mutations are a common phenomenon. Low rate of EGFR mutations and higher mutations in EGFR downstream pathways like those in TP53, HRAS, etc., suggest that anti EGFR strategies may not be very effective against OSCC, and there is need to identify more suitable targets.

\section{Conclusion}

The present study shows a higher incidence of mutations in tobacco-associated Indian OSCC, with presence of more than one mutation in most cases. Demonstration of downstream mutations in p53 and RAS provide evidences as to why the EGFR strategies are not effective in these patients, suggesting the role of combination of strategies or selection of strategies based on identifiable genetic mutations.

\section{Acknowledgement}

This study was adjudged as the best Indian Research at NATCON 2018 at Thiruvanathpuram, Kerala and was sent to SSO 2019 as Global poster. NB received a travelling fellowship from SSO to travel to San Diego and present the poster. The abstract of the study is published in Annals of Surgical Oncology 2019 (suppl). Batta N, Pandey M. Relation of cancer-related gene mutations and clinico-pathological factors in oral squamous cell carcinoma. Annals of Surgical Oncology 2019; 26(1suppl.): S207.

\section{Authors' contribution}

NB conducted the study, collected the data, did the statistical analysis and prepared the draft manuscript. MP conceived, designed and conducted the study, helped with the statistical analysis and edited the final draft of the manuscript. Both authors read and approved the final manuscript.

\section{Funding}

None

\section{Availability of data and materials}

Hard and soft copy of data is available with authors, and blinded data can be provided on reasonable requests within the provisions of Indian law.

\section{Ethics approval and consent to participate}

The study was approved by the Institute Ethics committee of IMS, BHU, and written informed consent was obtained from all participants.

\section{Consent for publication}

Not applicable as the study does not use individual patient data.

\section{Competing interests}

The authors declare that they have no conflict of interest.
Received: 3 August 2019 Accepted: 4 November 2019

Published online: 27 November 2019

\section{References}

1. Dikshit R, Gupta PC, Ramasundarahettige C, Gajalakshmi V, Aleksandrowicz L, Rajendra Badwe R, et al. Cancer mortality in India: a nationally representative survey. The Lancet. 2012;379:1807-16.

2. Khan, Z. An overview of oral cancer in Indian subcontinent and recommendations to decrease its incidence. Webmed Central Cancer 3 , WMC003626 (2012) http://www.webmedcentral.com/article_view/3626 (accessed August 2013).

3. Warnakulasuriya S. Global epidemiology of oral and oropharyngeal cancer. Oral Oncol. 2009:45:309-16.

4. World Health Organization. Tobacco free initiative http://www.who.int/ tobacco/research/cancer/en/ (accessed on 26 Oct 2013).

5. IARC Working Group on the Evaluation of Carcinogenic Risks to Humans. Betel-quid and areca-nut chewing and some areca-nut-derived nitrosamines. IARC monographs on the evaluation of carcinogenic risks to humans v. 85 Lyon, France, 2003 (http://monographs.iarc.fr/ENG/ Monographs/vol85/mono85.pdf) accessed 2013.

6. Boffetta P, Hecht S, Gray N, Gupta P, Strad K. Smokeless tobacco and cancer. Lancet Oncol. 2008;9:667-75.

7. Gupta PC, Ray CS, Sinha DN, Singh PK. Smokeless tobacco: a major public health problem in the SEA region: a review. Ind. J. Pub. Health. 2011;55:199-209.

8. Chocolatewala NM, Chaturvedi P. Role of human papilloma virus in the oral carcinogenesis: an Indian perspective. J. Can. Res. Ther 2009:5:71-7.

9. Vincenzi B, Zoccoli A, Pantano F, Venditti O, Galluzzo S. Cetuximab: from bench to bedside. Curr Cancer Drug Targets. 2010;10(1):80-95.

10. Stanton $\mathrm{P}$, et al. Epidermal growth factor receptor expression by human squamous cell carcinomas of the head and neck, cell lines and xenografts. Br J Cancer. 1994;70(3):427-33.

11. Bonner JA, et al. Radiotherapy plus cetuximab for squamous-cell carcinoma of the head and neck. N Engl J Med. 2006;354(6):567-78.

12. Vermorken JB, et al. Platinum-based chemotherapy plus cetuximab in head and neck Cancer. N Engl J Med. 2008;359(11):1116-27.

13. Licitra $\mathrm{L}$, et al. Predictive value of epidermal growth factor receptor expression for first-line chemotherapy plus cetuximab in patients with head and neck and colorectal cancer: analysis of data from the EXTREME and CRYSTAL studies. Eur J Cancer. 2013;49(6):1161-8.

14. Vermorken JB, et al. Open-label, uncontrolled, multicenter phase II study to evaluate the efficacy and toxicity of cetuximab as a single agent in patients with recurrent and/or metastatic squamous cell carcinoma of the head and neck who failed to respond to platinum-based therapy. J ClinOncol. 2007; 25(16):2171-7.

15. Vermorken JB, et al. Cisplatin and fluorouracil with or without panitumumab in patients with recurrent or metastatic squamous-cell carcinoma of the head and neck (SPECTRUM): an open-label phase 3 randomised trial. Lancet Oncol. 2013;14(8):697-710.

16. Eriksen JG, et al. OC-009: update of the randomised phase III trial DAHANCA 19: primary C-RT or RT and zalutumumab for squamous cell carcinomas of head and neck. RadiotherOncol. 2015;114:10.

17. Cowley GP, Smith JA, Gusterson BA. Increased EGF receptors on human squamous carcinoma cell lines. Br J Cancer. 1986;53(2):223-9.

18. Abdul Razak AR, et al. A phase II trial of dacomitinib, an oral pan-human EGF receptor (HER) inhibitor, as first-line treatment in recurrent and/or metastatic squamous-cell carcinoma of the head and neck. Ann Oncol. 2012;24(3):761-9.

19. Kim HS, et al. Phase II clinical and exploratory biomarker study of dacomitinib in patients with recurrent and/or metastatic squamous cell carcinoma of head and neck. Clin Cancer Res. 2015;21(3):544-52.

20. Ferris RL, et al. Nivolumabvs investigator's choice in recurrent or metastatic squamous cell carcinoma of the head and neck: 2-year long-term survival update of CheckMate 141 with analyses by tumor PD-L1 expression. OralOncol. 2018;81:45-51.

21. Ferris RL, et al. Nivolumab for recurrent squamous-cell carcinoma of the head and neck. N Engl J Med. 2016;375(19):1856-67.

22. Hsu C, et al. Safety and antitumor activity of pembrolizumab in patients with programmed death-ligand 1-positive nasopharyngeal carcinoma: results of the KEYNOTE-028. StudyJClinOncol. 2017;35:4050-6. 
23. Sina $M$, et al. P53 gene codon 72 polymorphism in patients with oral squamous cell carcinoma in the population of northern Iran. Med Oral Patol Oral Cir Bucal. 2014;19(6):e550-5.

24. Vlad, et al. High-risk TP53 mutations are associated with extra nodal extension in oral cavity squamous cell carcinoma. Clin Cancer Res. 2018; 24(7):1727-33.

25. Pickering $C R$, et al. Integrative genomic characterization of oral squamous cell carcinoma identifies frequent somatic drivers. Cancer Discov. 2013;3(7): 770-81.

26. Song et al. Common and complex Notch1 mutations in Chinese oral squamous cell carcinoma. Clin Cancer Res. 2014;1;20(3):701-710.

27. Boyle JO, et al. The incidence of p53 mutations increases with progression of head and neck cancer. Cancer Res. 1993;53(19):4477-80.

28. Lazarus $P$, et al. Relationship between p53 mutation incidence in oral cavity squamous cell carcinomas and patient tobacco use. Carcinogenesis. 1996; 17(4):733-9.

29. Ostwald C, et al. p53 mutational spectra are different between squamouscell carcinomas of the lip and the oral cavity. Int J Cancer. Int J Cancer. 2000;88(1):82-6.

30. Fadlullah $M Z$, et al. Genetically-defined novel oral squamous cell carcinoma cell lines for the development of molecular therapies. Oncotarget. 2016; 7(19):27802-18.

31. Okada $T$, lizuka N, Yamada-Okabe $H$, Mori N, Tamesa T, Takemoto N Tangoku A, Hamada K, Nakayama H, Miyamoto T, Uchimura S, Hamamoto Y, Oka M. Gene expression profile linked to p53 status in hepatitis C virus-related hepatocellular carcinoma. FEBS Lett. 2003;555(3): 583-90.

32. Parsons DW, Jones S, Zhang X, Lin JC, Leary RJ, Angenendt $P$, et al. An integrated genomic analysis of human glioblastoma multiforme. Science. 2008;321(5897):1807-12.

33. Dubot C, Bernard V, Sablin MP, Vacher S, Chemlali W, Schnitzler A, et al. Comprehensive genomic profiling of head and neck squamous cell carcinoma reveals FGFR1 amplifications and tumour genomic alterations burden as prognostic biomarkers of survival. Eur J Cancer. 2018;91:47-55.

34. Loeffler-Ragga J, et al. Low incidence of mutations in EGFR kinase domain in Caucasian patients with head and neck squamous cell carcinoma. European Journal of Cancer. 2006;42(1):109-11.

35. Bos JL. The ras gene family and human carcinogenesis. Mutation Research. 1988;195:255-71.

36. Michmerhuizen NL, Birkeland AC, Bradford CR, Brenner JC. Genetic determinants in head and neck squamous cell carcinoma and their influence on global personalized medicine. Genes Cancer. 2016;7(5-6): $182-200$.

37. Sathyan KM, Nalinakumari KR, Kannan S. H-Ras mutation modulates the expression of major cell cycle regulatory proteins and disease prognosis in oral carcinoma. Modern Pathology. 2007;20:1141-8.

38. Milasin, et al. High incidence of $\mathrm{H}$-ras oncogene mutations in squamous cell carcinoma of lip vermilion. J Oral Pathol Med. 1994;23:298-301.

39. Das N, Majumder J. UB Dasgupta. Ras gene mutations in oral cancer in eastern India Oral Oncology. 2000;36:76-80.

40. Weber $A$, et al. Mutations of the BRAF gene in squamous cell carcinoma of the head and neck. Oncogene. 2003;22:4757-9.

41. Karl C, Bruckman, et al. Mutational analyses of the BRAF, KRAS, and PIK3CA genes in oral squamous cell carcinoma. Oral Surg Oral Med Oral Pathol Oral RadiolEndod. 2010;110:632-7.

42. Lim, et al. Differential mechanisms of CDKN2A (p16) alteration in oral tongue squamous cell carcinomas and correlation with patient outcome. Int. J. Cancer. 2014;135:887-95.

43. Samuels $Y$, Wang $Z$, Bardelli $A$, et al. High frequency of mutations of the PIK3CA gene in human cancers. Science. 2004;304:554.

44. Kozaki, et al. PIK3CA mutation is an oncogenic aberration at advanced stages of oral squamous cell carcinoma. Cancer Sci. 2006;97:1351-8.

45. Chen S, Liu H, Liao C, Huang P, Huang Y, Hsu A, et al. Ultra-deep targeted sequencing of advanced oral squamous cell carcinoma identifies a mutation-based prognostic gene signature. Oncotarget. 2015;6(20):1866-80.

46. Feldman R, Gatalica Z, Knezetic J, Reddy S, Nathan C, Javadi N, Teknos T. Molecular profiling of head and neck squamous cell carcinoma. Head \& Neck. 2016:E1625-38.

47. Lui WWY, Hedberg ML, Li H, et al. Frequent mutation of the PI3K pathway in head and neck cancer defines predictive biomarkers. Cancer Discovery. 2013;3:761-9.
48. Inokuchi K, Yamaguchi $\mathrm{H}$, Tarusawa M, et al. Abnormality of c-kit oncoprotein in certain patients with chronic myelogenousleukemia-potential clinical significance. Leukemia. 2002;16:170-7.

49. Tan, et al. Tonque carcinoma infrequently harbor common actionable genetic alterations. BMC Cancer. 2014;14:679.

50. Chung CH, Guthrie VB, Masica DL, Tokheim C, Kang H, Richmon J, Agrawal N, Fakhry C, Quon H, Subramaniam RM, Zuo Z, Seiwert T, Chalmers ZR, Frampton GM, Ali SM, Yelensky R, Stephens PJ, Miller VA, Karchin R, Bishop JA.Genomic alterations in head and neck squamous cell carcinoma determined by cancer gene-targeted sequencing. Ann Oncol. 2015;26(6): 1216-1223. doi: https://doi.org/10.1093/annonc/mdv109. Epub 2015 Feb 23.

51. Stransky N, Egloff AM, Tward AD, Kostic AD, Cibulskis K, Sivachenko A, Kryukov GV, Lawrence MS, Sougnez C, McKenna A, Shefler E, Ramos AH, Stojanov P, Carter SL, Voet D, Cortés ML, Auclair D, Berger MF, Saksena G, Guiducci C, Onofrio RC, Parkin M, Romkes M, Weissfeld JL, Seethala RR, Wang L, Rangel-Escareño C, Fernandez-Lopez JC, Hidalgo-Miranda A, Melendez-Zajgla J, Winckler W, Ardlie K, Gabriel SB, Meyerson M, Lander ES, Getz G, Golub TR, Garraway LA, Grandis JR. The mutational landscape of head and neck squamous cell carcinoma. Science. 2011;333(6046):1157-60. https://doi.org/10.1126/science.1208130 Epub 2011 Jul 28.

52. Agrawal N, Frederick MJ, Pickering CR, Bettegowda C, Chang K, Li RJ, Fakhry C, Xie TX, Zhang J, Wang J, Zhang N, El-Naggar AK, Jasser SA, Weinstein JN, Treviño L, Drummond JA, Muzny DM, Wu Y, Wood LD, Hruban RH, Westra WH, Koch WM, Califano JA, Gibbs RA, Sidransky D, Vogelstein B, Velculescu VE, Papadopoulos N, Wheeler DA, Kinzler KW, Myers JN. Exome sequencing of head and neck squamous cell carcinoma reveals inactivating mutations in NOTCH1. Science. 2011;333(6046):1154-1157. doi: https://doi.org/10.1126/ science.1206923. Epub 2011 Jul 28.

\section{Publisher's Note}

Springer Nature remains neutral with regard to jurisdictional claims in published maps and institutional affiliations.

Ready to submit your research? Choose BMC and benefit from:

- fast, convenient online submission

- thorough peer review by experienced researchers in your field

- rapid publication on acceptance

- support for research data, including large and complex data types

- gold Open Access which fosters wider collaboration and increased citations

- maximum visibility for your research: over $100 \mathrm{M}$ website views per year

At $\mathrm{BMC}$, research is always in progress.

Learn more biomedcentral.com/submissions 